\title{
Totally Laparoscopic Anatomical Hepatectomy Exposing the Major Hepatic Veins from the Root Side: a Case of the Right Anterior Sectorectomy (with Video)
}

\author{
Goro Honda • Masanao Kurata • Yukihiro Okuda • \\ Shin Kobayashi • Katsunori Sakamoto • \\ Keiichi Takahashi
}

Received: 2 February 2014 / Accepted: 6 May 2014 / Published online: 20 May 2014

(C) 2014 The Author(s). This article is published with open access at Springerlink.com

\begin{abstract}
Laparoscopic hepatectomy has rapidly evolved recently; ${ }^{1-5}$ however, laparoscopic anatomical hepatectomy has yet to become widely used, although anatomical hepatectomy is ideal, especially for curative treatment of hepatocellular carcinoma, and is widely accepted via open approach. ${ }^{6-10}$ This is because good-experienced skills, for example, exposing Glissonean pedicles and hepatic veins on the cutting plane, are required in order to perform anatomical hepatectomy via a pure laparoscopic approach. We obtained good results for various totally laparoscopic anatomical hepatectomies using the standardized techniques. We exposed the major hepatic veins from the root side by utilizing the unique view from the caudal side in the laparoscopic approach, and moved CUSA from the root side toward the peripheral side to avoid splitting the bifurcation of the hepatic vein. ${ }^{11-13}$ We performed totally laparoscopic anatomical hepatectomy for 47 patients from August, 2008, to December, 2012 (Table 1). In most types of anatomical hepatectomy, the mean blood loss was $<500 \mathrm{ml}$. Conversion to open surgery was required in two patients. Postoperative complications were prolonged ascites in two, peroneal palsy in two, and biloma in one. Mortality was zero. The embedded video demonstrates totally laparoscopic right anterior sectorectomy. In conclusion, our standardized techniques make laparoscopic anatomical hepatectomy more feasible.
\end{abstract}

Keywords Laparoscopic hepatectomy · Anatomical hepatectomy $\cdot$ CUSA
This is a multimedia article. Please refer to the video included as a Supplementary Material.

This paper was presented in Annual clinical congress of American College of Surgeons on 9th October, 2013 at Washington DC.

Electronic supplementary material The online version of this article (doi:10.1007/s11605-014-2538-9) contains supplementary material, which is available to authorized users.

G. Honda $(\bowtie) \cdot$ M. Kurata $\cdot$ Y. Okuda $\cdot$ S. Kobayashi $\cdot$

K. Sakamoto $\cdot$ K. Takahashi

Department of Surgery, Tokyo Metropolitan Cancer and Infectious

Diseases Center Komagome Hospital, 3-18-22 Honkomagome,

Bunkyo-ku 113-8677, Tokyo, Japan

e-mail: ghon@cick.jp 
Table 1 The result of 47 patients who underwent totally laparoscopic anatomical hepatectomy

\begin{tabular}{|c|c|c|c|c|c|c|}
\hline & $\begin{array}{l}\text { Number } \\
\text { of cases }\end{array}$ & Time (mean) & $\begin{array}{l}\text { Blood loss } \\
\text { (mean) }\end{array}$ & Additional procedures & $\begin{array}{l}\text { Conversion to open } \\
\text { surgery }\end{array}$ & Complications \\
\hline Rt. Hemi. & 4 & $6 \mathrm{hr} .10 \mathrm{~min}$. & $270 \mathrm{~g}$ & Colectomy $\times 1$, Stoma closure $\times 1$ & 0 & \\
\hline Lt. Hemi. & 4 & $5 \mathrm{hr} .06 \mathrm{~min}$. & $246 \mathrm{~g}$ & S5 partial $\times 1$ & 0 & \\
\hline Rt. Ant. Sector & 5 & $7 \mathrm{hr} .03 \mathrm{~min}$. & $596 \mathrm{~g}$ & & 0 & Ascites $\times 2$ \\
\hline Rt. Post. Sector & 7 & 7 hr. 32 min. & $382 \mathrm{~g}$ & S8 partial $\times 2$, Rt.adrenectomy $\times 1$ & 0 & Peroneal palsy $\times 1$ \\
\hline Lt. lateral Sector & 7 & $3 \mathrm{hr} .29 \mathrm{~min}$. & $211 \mathrm{~g}$ & & 0 & \\
\hline Lt. Medial Sector & 4 & $5 \mathrm{hr} .10 \mathrm{~min}$. & $310 \mathrm{~g}$ & $\mathrm{~S} 8$ partial $\times 1$ & 0 & \\
\hline Dorsal Rt. Ant. Segment & 1 & $6 \mathrm{hr} .35 \mathrm{~min}$. & $395 \mathrm{~g}$ & & 0 & Peroneal palsy $\times 1$ \\
\hline S2 (segmentectomy) & 1 & $7 \mathrm{hr} .15 \mathrm{~min}$. & $310 \mathrm{~g}$ & S4 partial & 0 & \\
\hline S3 (segmentectomy) & 1 & $3 \mathrm{hr} .22 \mathrm{~min}$. & $5 \mathrm{~g}$ & & 0 & \\
\hline S5 (segmentectomy) & 3 & $6 \mathrm{hr} .28 \mathrm{~min}$. & $262 \mathrm{~g}$ & & 0 & \\
\hline S6 (segmentectomy) & 4 & $5 \mathrm{hr} .00 \mathrm{~min}$. & $140 \mathrm{~g}$ & & 0 & \\
\hline S5+6 (segmentectomy) & 2 & $8 \mathrm{hr} .14 \mathrm{~min}$. & $765 \mathrm{~g}$ & & 0 & \\
\hline S8 (segmentectomy) & 2 & $8 \mathrm{hr} .00 \mathrm{~min}$. & $795 \mathrm{~g}$ & & excessive time $\times 2$ & \\
\hline Rt. Caudate lobe & 2 & $8 \mathrm{hr} .51 \mathrm{~min}$. & $240 \mathrm{~g}$ & S2 partial \& Coloctomy $\times 1$ & 0 & Biloma $\times 1$ \\
\hline
\end{tabular}

Open Access This article is distributed under the terms of the Creative Commons Attribution License which permits any use, distribution, and reproduction in any medium, provided the original author(s) and the source are credited.

\section{References}

1. Dagher I, O'Rourke N, Geller DA, Cherqui D, Belli G, Gamblin TC, Lainas P, Laurent A, Nguyen KT, Marvin MR, Thomas M, Ravindra K, Fielding G, Franco D, Buell JF. Laparoscopic Major Hepatectomy: An Evolution in Standard of Care. Ann Surg 2009;250:856-860.

2. Han HS, Cho JY, Yoon YS. Techniques for performing laparoscopic liver resection in various hepatic locations. J Hepatobiliary Pancreat Surg 2009;16:427-432.

3. Abu Hilal M, Di Fabio F, Abu Salameh M, Pearce NW. Oncological efficiency analysis of laparoscopic liver resection for primary and metastatic cancer: a single-center UK experience. Arch Surg 2012;147:42-48.

4. Kaneko H, Takagi S, Otsuka Y, Tsuchiya M, Tamura A, Katagiri T, Maeda T, Shiba T. Laparoscopic liver resection of hepatocellular carcinoma. Am J Surg 2005;189:190-194.

5. Lin NC, Nitta H, Wakabayashi G. Laparoscopic major hepatectomy: a systematic literature review and comparison of 3 techniques. Ann Surg 2013;257:205-213

6. Kobayashi A, Miyagawa S, Miwa S, Nakata T. Prognostic impact of anatomical resection on early and late intrahepatic recurrence in patients with hepatocellular carcinoma. J Hepatobiliary Pancreat Surg 2008;15:515-521.

7. Eguchi S, Kanematsu T, Arii S, Okazaki M, Okita K, Omata M, Ikai I, Kudo M, Kojiro M, Makuuchi M, Monden M, Matsuyama Y, Nakanuma Y, Takayasu K; Liver Cancer Study Group of Japan. Comparison of the outcomes between an anatomical subsegmentectomy and a non-anatomical minor hepatectomy for single hepatocellular carcinomas based on a Japanese nationwide survey. Surgery 2008;143:469-475.

8. Yamamoto J, Kosuge T, Takayama T, Shimada K, Yamasaki S, Ozaki H, Yamaguchi N, Makuuchi M. Recurrence of hepatocellular carcinoma after surgery. Br J Surg 1996;83:1219-1222.

9. Regimbeau JM, Kianmanesh R, Farges O, Dondero F, Sauvanet A, Belghiti J. Extent of liver resection influences the outcome in patients with cirrhosis and small hepatocellular carcinoma. Surgery 2002;131:311-317.

10. Sakon M, Nagano H, Nakamori S, Dono K, Umeshita K, Murakami T, Nakamura H, Monden M. Intrahepatic recurrences of hepatocellular carcinoma after hepatectomy: Analysis based on tumor hemodynamics. Arch Surg 2002;137:94-99.

11. Honda G, Kurata M, Okuda Y, Kobayashi S, Tadano S, Yamaguchi T, Matsumoto H, Nakano D, Takahashi K. Totally laparoscopic hepatectomy exposing the major vessels. J Hepatobiliary Pancreat Sci 2013;20:435-440.

12. Okuda Y, Honda G, Kurata M, Kobayashi S, Sakamoto K. Dorsal approach to the middle hepatic vein in laparoscopic left hemihepatectomy. J Am Coll Surg 2014. doi:10.1016/j.jamcollsurg.2014.01.068.

13. Okuda Y, Honda G, Kurata M, Kobayashi S. A useful and convenient procedure for intermittent vascular occlusion in laparoscopic hepatectomy. Asian J Endosc Surg 2013;6:100-103. 